\title{
Bound states and lifetime of an electron on a bulk helium surface
}

\author{
M. H. Degani, ${ }^{1, *}$ G. A. Farias, ${ }^{1, \dagger}$ and F. M. Peeters ${ }^{2, *}$ \\ ${ }^{1}$ Departamento de Física, Universidade Federal do Ceará, Caixa Postal 6030, Campus do Pici, 60455-760 Fortaleza, Ceará, Brazil \\ ${ }^{2}$ Department of Physics (U213), University of Antwerp (Campus Middelheim), Groenenborgerlaan 171, B-2020 Antwerpen, Belgium
}

(Received 1 April 2005; published 6 September 2005)

\begin{abstract}
We propose an effective potential for an excess electron near the helium liquid-vapor interface that takes into account the diffuseness of the liquid-vapor interface and the classical image potential. The splitting of the first two excited states of the excess electron bound to the helium liquid-vapor interface as a function of an external constant electric field applied perpendicular to the interface is in excellent agreement with recent experiments. The effect of a parallel magnetic field on the energy levels are calculated. Single-electron tunneling of the electron out of its surface state is studied as a function of the electric field applied to the system. We found that the tunneling time has a linear dependence on the electric field.
\end{abstract}

DOI: 10.1103/PhysRevB.72.125408

PACS number(s): 73.20.At

\section{INTRODUCTION}

Sommer ${ }^{1}$ pointed out that an electron could be bound to the surface of liquid helium. Cole and Cohen ${ }^{2}$ and Shikin ${ }^{3}$ independently showed that an electron could form a surface state outside some liquids due to the negative electron affinity preventing the electron from entering the liquid and the classical image force attracting the electron to the helium surface. Since then, an enormous theoretical and experimental effort has been dedicated to the understanding of the electronic properties of electrons on a liquid helium surface in several situations, ${ }^{4-6}$ such as the presence of external fields. The presence of an electric field and a transverse magnetic field, perpendicular and parallel to the vacuum-liquid interface, respectively, changes considerably the surface states. An applied electric field pushes the electron towards the helium surface. However, if the electric field is applied in the opposite direction, metastable states can occur. ${ }^{7}$ In addition, the existence of a transverse magnetic field can modify significatively the tunneling of the electron out of the surface state. $^{8}$

The main approximation used in order to calculate the surface electronic states is that the electron is subject to a competition of a short-range repulsion, which arises from the requirement of the Pauli exclusion principle and is represented by a large potential step that prevents the electron penetration into the liquid, and a long-range attraction due to the polarization potential, represented by the classical image potential. Cole ${ }^{9}$ proposed a model for the effective potential, which avoided the divergence of the image potential by introducing a cutoff at short distances of the order of the interatomic spacing in the liquid. Grimes et al. ${ }^{11}$ and Hipólito et $a l .{ }^{12}$ introduced a parameter that shifted the divergence of the image potential inside the liquid; the value of this shift was obtained by fitting the difference between the ground state and the excited state to the experimental results. Stern ${ }^{13}$ proposed a model including a gradual transition between the two dielectrics. Rama Krishna et al. ${ }^{14}$ calculated the binding energy of an electron attached to the surface of helium clusters, and liquid helium, using a potential that consisted of a shortrange electron-helium pseudopotential and a long-range polarization potential. More recently, Cheng et al. ${ }^{15}$ used a
Hartree-type of effective potential that takes into account the nonuniform liquid density profile. Ancilotto and Toigo ${ }^{16}$ also calculated the properties of an electron inside the helium liquid and considered a nonuniform liquid density.

Motivated by recent experiments ${ }^{17}$ on the temperature dependence of the transition energies between the bound states of the electron at the helium/vacuum interface, we revisit these problems by proposing a simple potential that describes the electron-helium liquid interaction. We take into account the temperature-dependent displacement of the liquid helium-vapor interface, as calculated by Cole, ${ }^{9}$ which is a measure of the diffuseness of the helium barrier. From this, we build the electron-helium surface interaction potential, avoiding the divergence at the vacuum-liquid $\mathrm{He}$ interface. Contrary to previous theoretical studies, there are no free fitting parameters in our model. Including an external electric and transverse magnetic fields, we calculated the energy of the ground and first excited states. Excellent agreement with the experiments of Grimes et al. ${ }^{11}$ and with the recent results of Collin et al. ${ }^{17}$ for the electric field dependence of the transitions between the ground state and the two first excited states is found for different temperatures.

This paper is organized as follows. In Sec. II, we discuss the proposed potential model and the method used to solve the Schrödinger equation. In Sec. III, we present the effects of an external electric field applied perpendicular to and a magnetic field applied parallel to the helium surface, respectively, on the electronic levels. In Sec. IV, the probability for electron escape when an electric and/or a magnetic field is applied to the system is calculated as a function of time. The electron average position is also calculated as a function of time, and from this behavior, the effective mass is inferred. Finally, in Sec. V, we present our conclusions.

\section{HAMILTONIAN MODEL}

The Hamiltonian for the electron surface state, including external electric and magnetic fields, applied perpendicular and parallel to the interface, respectively, can be written in the following way: 


$$
H(z)=-\frac{\hbar^{2}}{2 m} \frac{\partial^{2}}{\partial z^{2}}+V(z),
$$

with the potential given by

$$
V(z)=V_{\text {eff }}(z)+V_{\text {field }}(z),
$$

where the first term corresponds to the contribution of the barrier potential, which limits the electron penetration to the helium and the classical image potential, $V_{\text {eff }}(z)=V_{b}(z)$ $+V_{i m}(z)$. The last term in Eq. (2) is related to the presence of electric and magnetic fields, $V_{\text {field }}(z)=V_{e l}(z)+V_{\text {mag }}(z)$. For the barrier potential, we propose the function

$$
V_{b}(z)=\frac{V_{0}}{\exp (z / \delta)+1},
$$

where $V_{0}$ is the negative of the electron affinity, which is equal to $1 \mathrm{eV},{ }^{1}$ and $\delta=\Delta z / 4$, where $\Delta z$ is the surface thickness. The latter was calculated by $\mathrm{Cole}^{9}$ as a function of temperature using the hydrodynamic approximation. The image potential is taken to be

$$
-V_{i m}(z)=\theta(z-\delta) \frac{Q e^{2}}{z},
$$

following the model considered by Grimes et al.,${ }^{11}$ Cole, ${ }^{10}$ and Hipólito et al., ${ }^{12}$ with $Q=\left(\varepsilon_{\mathrm{He}}-1\right) /\left(\varepsilon_{\mathrm{He}}+1\right)$ and $\varepsilon_{\mathrm{He}}$ $=1.05723$ the helium dielectric constant. The contribution due to the presence of an external electric field applied perpendicular to the helium surface is given by

$$
V_{e l}(z)= \pm e F z
$$

with the sign $+(-)$ associated to the field that tends to press (pull) the electron to (from) the helium interface. The magnetic field parallel to the interface produces a potential given by

$$
V_{\text {mag }}(z)=m w_{c}^{2}\left(z-z_{0}\right)^{2} / 2,
$$

where $w_{c}=e B_{\|} / m_{0} c$ is the cyclotron frequency; $m_{0}$ the electron mass; and $z_{0}=p_{y} / w_{c} m_{0}$, with $p_{y}$ the canonical momentum, which is a constant of motion in the Landau gauge.

The method used to calculate the electronic states, within the effective mass approximation, is based on the solution of the time-dependent Schrödinger equation ${ }^{18-20}$

$$
i \hbar \frac{\partial}{\partial t} \Psi(\vec{r}, t)=H \Psi(\vec{r}, t) .
$$

The evolution method is not limited to small perturbations and can be used for nonlinear effects, including extreme conditions created by laser pulses. Another advantage in using this method is that only states of interest are involved and the calculation can be made to scale linearly with the size of the system, contrary to methods based on the linear response formalism, which involves diagonalization of large matrices. In general, it is not possible to perform the exponentiation of an operator exactly, and one must bring the operators to a diagonal form. The time evolution after one time step $\Delta t$ is given by

$$
\Psi(\vec{r}, t+\Delta t)=e^{-i H \Delta t / \hbar} \Psi(\vec{r}, t) .
$$

Since the operator is unitary, the normalization of the wave function is preserved and thus guarantees the conservation of the probability and the unconditional stability of the method. The time evolution of the wave function [Eq. (8)] can be written as

$\Psi(\vec{r}, t+\Delta t)=e^{-i V(\vec{r}) \Delta t / 2 \hbar} e^{-i p^{2} \Delta t / 2 \hbar m^{*}} e^{-i V(\vec{r}) \Delta t / 2 \hbar} \Psi(\vec{r}, t)+O\left(\Delta t^{3}\right)$.

The error introduced in this expression by dropping the term $O\left(\Delta t^{3}\right)$ results from the fact that kinetic and potential operators do not commute. When we study systems with periodic boundary conditions, the exponential containing the kinetic operator is efficiently treated by the fast Fourier transform, since it is diagonal in reciprocal space. However, when the system is not periodic, we use the following expansion of the exponential (e.g., see Crank-Nicholson ${ }^{21}$ ):

$$
e^{-i p^{2} \Delta t / 2 \hbar m^{*}} \cong\left[1-i \frac{\hbar \Delta t}{4 m^{*}} \frac{\partial^{2}}{\partial x^{2}}\right]^{-1}\left[1+i \frac{\hbar \Delta t}{4 m^{*}} \frac{\partial^{2}}{\partial x^{2}}\right],
$$

which is correct up to the order $O\left(\Delta t^{3}\right)$. The derivatives are calculated as finite differences such that the solution is reduced to the inversion of a tridiagonal matrix. For a timeindependent Hamiltonian, it is time-reversal invariant and conserves the total energy exactly. In order to obtain the eigenstates of a time-independent Hamiltonian, the scheme described above is applied to an initial wave packet and the evolution is made in the imaginary time domain; that is, one substitutes $t$ by $-i \tau$. After a few steps, the wave function converges to the ground state of the system. The excited states are obtained using the same procedure, in combination with a Gram-Schmidt orthonormalization.

\section{SURFACE STATE ENERGIES}

Figure 1 shows our potential $V_{\text {eff }}(z)$ in the absence of any external fields, which describes the electron-helium surface interaction. Notice that it has no divergence at $z=0$ and is temperature dependent. The latter enters through the parameter $\Delta z$, which is the surface displacement as calculated by Cole $^{9}$ within the hydrodynamic approximation. Since our model potential depends implicitly on temperature, we present $V_{\text {eff }}(z)$ in Fig. 1 for several temperatures.

Notice that the depth of the potential is strongly reduced with increasing temperature and that the effective position of the helium surface moves to positive $z$. To calculate the energy, we discretized the system in an interval of $6000 \AA$ using a grid with 15000 equally spaced points. The initial wave functions were prepared using Gaussian functions and the imaginary time propagation was used. After a few time steps $\Delta t$, the system has converged to the eigenstates of the system. One of the advantages of the present method is that it is only weakly dependent on the initial wave function. We show in Fig. 2 the electron excitation energy from the ground state to the first and second excited states, as a function of the external electric field applied perpendicular to the helium surface, for two values of the helium liquid temperature: $T$ $=1.0 \mathrm{~K}$ (solid line) and $T=1.3 \mathrm{~K}$ (dotted curves). For the temperature $T=1.0 \mathrm{~K}$, our results are in very good agreement 


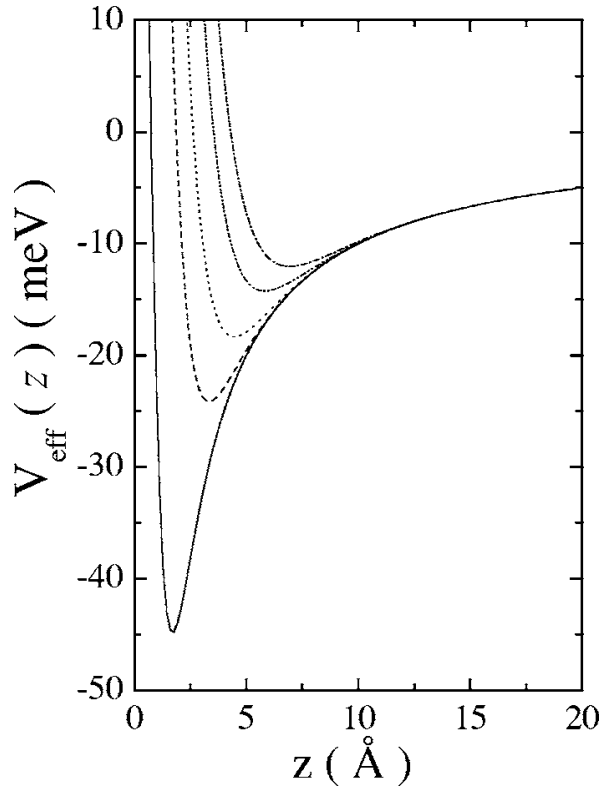

FIG. 1. Effective potential for temperatures. $T=0 \mathrm{~K}$ (solid), $0.5 \mathrm{~K}$ (dashed), $1.0 \mathrm{~K}$ (dotted), $1.5 \mathrm{~K}$ (dashed-dotted-dotted), and $2.0 \mathrm{~K}$ (dashed-dotted) respectively.

with those obtained experimentally by Collin et al. ${ }^{17}$ for transitions between the ground state and the first excited state. Increasing the temperature, we expect that the ground state energy increases more than the two excited states and consequently the excitation energies should decrease. The experimental results obtained by Grimes et al. ${ }^{11}$ at $1.2 \mathrm{~K}$,

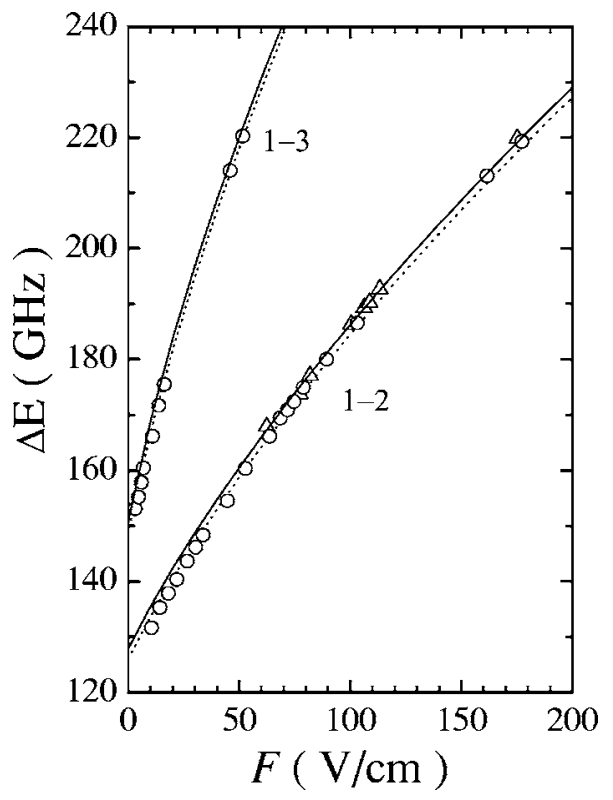

FIG. 2. Transition energy between ground state and first (1-2) and second excited states (1-3), respectively, as a function of an external electric field applied perpendicular to the helium surface, for two values of the helium liquid temperature, $T=1.0 \mathrm{~K}$ (solid line) and $T=1.3 \mathrm{~K}$ (dotted line). The experimental results obtained by Grimes et al. (see Ref. 11) at $T=1.2 \mathrm{~K}$ (circles), and by Collin et al. (see Ref. 17) at $T=1.0 \mathrm{~K}$ (triangles), respectively, are also shown. extrapolated to zero field give $125.9 \pm 0.2$ and $148.6 \pm 0.3 \mathrm{GHz}$ for transitions from the ground state to the first and second states, respectively. Our model gives 126.0 and $148.8 \mathrm{GHz}$ for those transitions at $T=1.3 \mathrm{~K}$. Again, an excellent agreement between experiment and theory is obtained. The proposed effective potential is deeper for lower temperature (see Fig. 1), because the diffuseness of the interface becomes smaller, and results in an enhanced splitting between the states. For $T=1.2 \mathrm{~K}$, the disagreement between our results and the experimental data ${ }^{11}$ is within $0.6 \%$.

Notice also that the results of our model are in agreement with the experimental data over the whole range of applied electric fields and that it contains no adjustable parameter. This is in contrast to the variational model proposed by Grimes et al., ${ }^{11}$ where at high fields the calculated energy differences deviate from the measured values. Rama Krishna $^{14}$ and Cheng et al., ${ }^{15}$ present results only for zero electric field. In Table I we collected the principal theoretical results obtained by different authors using adjustable parameters to fit the transitions to the experimental values and compared them with the present results.

In Fig. 3 we present the temperature dependence of the transition energy between the ground state and the first (1-2) and second excited states (1-3), respectively, for zero field. The full triangles are the results at $T=1.2 \mathrm{~K}$, in agreement with the extrapolated experimental results of Grimes et al. ${ }^{11}$ for zero field. These results are consistent with the ones presented in Fig. 2, where the temperature decreases the transition energies. This is a consequence of the fact that the ground state energy increases more rapidly with the temperature than the two excited states.

In Fig. 4 we show the average position $z_{a v}$ of the electron above the helium interface, for the ground, first, and second excited states, respectively, as a function of the applied external electric field, at the temperature $T=1.3 \mathrm{~K}$. As can be seen, $z_{a v}$ decreases with pressing electric field for all three states, as expected. The decrease is more pronounced for the excited states, which are less bound and more extended.

To analyze the effect of an external magnetic field applied parallel to the helium surface, we show in Fig. 5 the transition energy between the ground state and the first (1-2) and second excited states (1-3), respectively, as a function of the magnetic field, for two values of the temperature: $T=0 \mathrm{~K}$ (solid line) and $T=1.3 \mathrm{~K}$ (dotted line), in the absence of an external electric field. The change in the potential with temperature is more significant on the ground state and consequently the transition energies decrease with temperature, but this effect is small compared with the changes due to the presence of an external electric field (see Fig. 2) or a parallel magnetic field (Fig. 5). In Fig. 6 we show the average displacement $z_{a v}$ from the helium interface, as a function of the in-plane magnetic field, for the ground state, the first excited state, and the second excited state, in the absence of an external electric field, for two values of the temperature: $T$ $=0 \mathrm{~K}$ (solid line) and $T=1.3 \mathrm{~K}$ (dotted line). The magnetic field results in an additional localization potential for the electron and consequently we expect that the displacement decreases with field. As observed for the excitation energies, the temperature dependence of the average displacement is also small. In Fig. 7 we show the dispersion relation of the 
TABLE I. Electron excitation energy from the ground state to the first $\Delta E_{12}$ and second excited state $\Delta E_{13}$ in the absence of external fields: experimental results of Grimes et al. (see Ref. 11) (A); Hydrogenic model (B); Stern (see Ref. 13) (C); Rama Krishna (see Ref. 14) (D); Cheng et al. (see Ref. 15) (E); This work at $T=1.2 \mathrm{~K}(\mathrm{~F})$ and $T=1.3 \mathrm{~K}(\mathrm{G})$ respectively.

\begin{tabular}{cccccccc}
\hline \hline & $\mathrm{A}$ & $\mathrm{B}$ & $\mathrm{C}$ & $\mathrm{D}$ & $\mathrm{E}$ & $\mathrm{F}$ & $\mathrm{G}$ \\
\hline$\Delta E_{12}(\mathrm{GHz})$ & $125.9 \pm 0.2$ & 132.3 & 125.5 & 125.0 & 122.1 & 126.6 & 126.0 \\
$\Delta E_{13}(\mathrm{GHz})$ & $148.6 \pm 0.3$ & 156.5 & 148.3 & 148.3 & 144.4 & 149.5 & 148.8 \\
\hline \hline
\end{tabular}

electron, as a function of the momentum parallel to the helium surface $K_{y}$, in the presence of an in-plane magnetic field $\left(B_{\|}=1.5 \mathrm{~T}\right)$, for the ground state, first excited state, and second excited state, in the absence of an external electric field, at $T=1.3 \mathrm{~K}$. In the absence of an in-plane magnetic field, this energy dispersion is a single parabola $\hbar^{2} k_{y}^{2} / 2 m$ for all the electron bound states. Taking the second derivative of this dispersion relation we obtained the electron effective mass parallel to the liquid surface, for the ground state $m_{1}^{\|}$ $=1.022 m_{e}$, first excited state $m_{2}^{\|}=1.105 m_{e}$, and second excited state $m_{3}^{\|}=1.143 m_{e}$, where $m_{e}$ is the free electron mass.

\section{TUNNELING PROCESS}

Up to now we considered only external electric fields that press the electron to the helium surface and studied the stable ground and excited states. In this section we will analyze the electron behavior as a function of time, when it escapes from the surface due to an electric and/or a magnetic field applied to the system. We consider now the case in which the electric field is applied along the $z$ direction such that it pulls the electron away from the helium surface. There is now a competition between the attractive effective potential and the repulsive electric field potential, which ultimately will favor

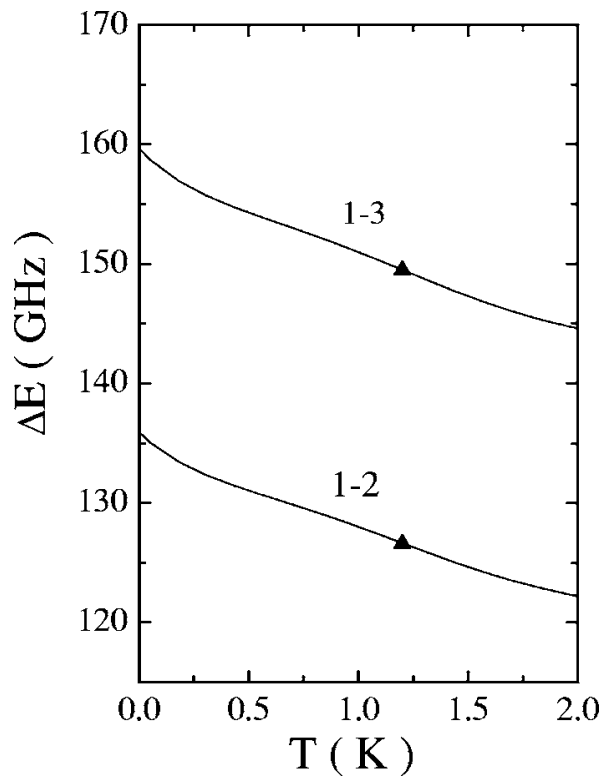

FIG. 3. Transition energy between ground state and first (1-2) and second excited state (1-3), respectively, as a function of the temperature, in the absence of external fields. The full triangles are the experimental results at $T=1.2 \mathrm{~K}$. the electron to tunnel out of its bound state. There is a nonzero probability that the electron will tunnel through the potential barrier separating the bound state and the unbound state at $z=0$. In order to study the ionization of an electron bound to the He surface, we start with initial electron states as obtained at zero electric field and use the time evolution [Eq. (8)] to investigate the electric field induced tunneling towards an unbound state. Let us now define the tunneling probability as

$$
P(t)=1-R(t)
$$

where $R(t)=\left|\left\langle\Psi(\vec{r}, 0) \mid \Psi^{F}(\vec{r}, t)\right\rangle\right|^{2}$, with $\Psi^{F}(\vec{r}, t)$ the wave function at time $t$ in the presence of the electric field, and the tunneling time is being defined as, the smallest $t$ for which $P(t)=1$. In Fig. 8 , we present the tunneling probability as a function of time for the three lowest states, in the presence of an external electric field of $F=300 \mathrm{~V} / \mathrm{cm}$. As expected, the excited states are ionized more quickly than the ground state, since they are less strongly bound. Another important physical quantity was calculated: electron average position from the interface $z_{a v}$ as a function of time, which is shown in Fig. 9 for $T=1.0 \mathrm{~K}$ and $F=300 \mathrm{~V} / \mathrm{cm}$. These parabolic-like curves show an accelerated motion from which it is possible

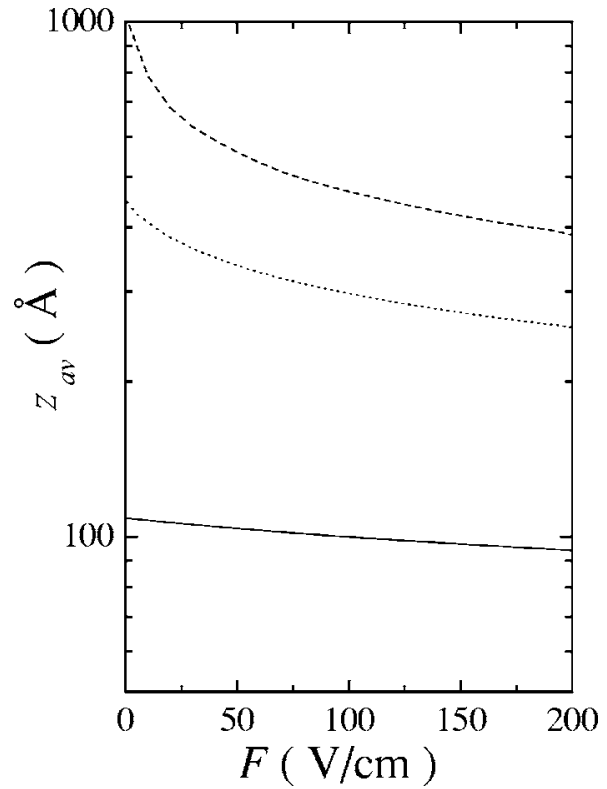

FIG. 4. Average displacement $z_{a v}$ from the interface as a function of the applied external electric field. Ground state (solid line), first excited state (dotted line), and second excited state (dashed line) for $T=1.3 \mathrm{~K}$. 


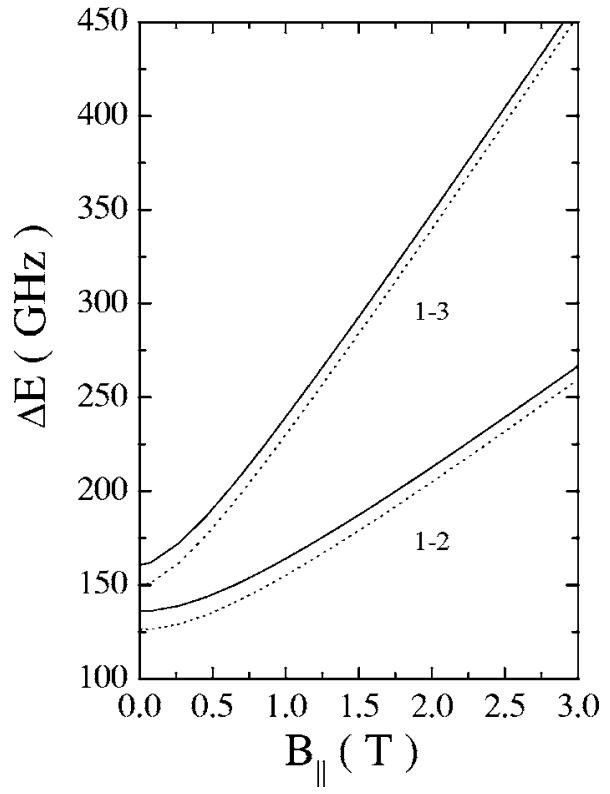

FIG. 5. Transition energy between the ground state and first (1-2) and second excited states (1-3), for $T=0 \mathrm{~K}$ (dotted line) and $T=1.3 \mathrm{~K}$ (solid line), respectively, as a function of the external parallel magnetic field, in the absence of an external electric field.

to extract the electron effective mass in the perpendicular direction with respect to the helium surface. For the considered parameters, we found $m_{1}^{\perp}=1.188 m_{e}, m_{2}^{\perp}=1.059 m_{e}$, and $m_{3}^{\perp}=1.028 m_{e}$, for the ground state, first excited state, and second excited state, respectively. Notice that these perpendicular masses decrease with increasing excitation state, which is opposite to the parallel masses found in the previ-

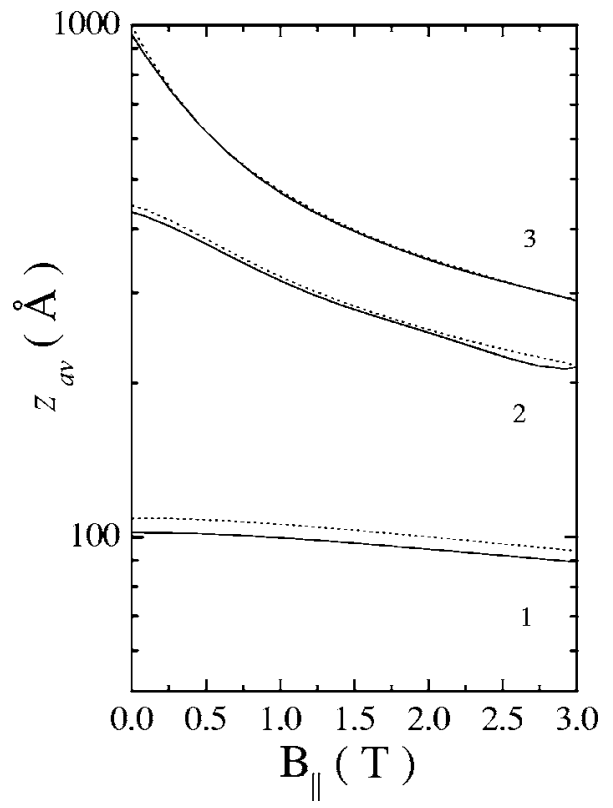

FIG. 6. Average displacement $z_{a v}$ from the helium interface, as a function of the external in-plane magnetic field, for the ground state (1), first excited state (2), and second excited state (3), in the absence of an external electric field. Solid line (dotted line) corresponds to a helium temperature of $T=0 \mathrm{~K}(T=1.3 \mathrm{~K})$.

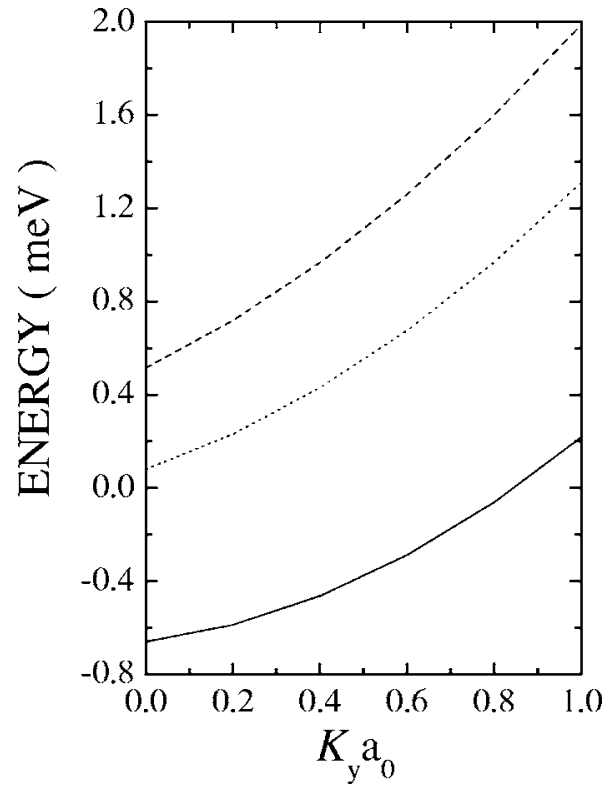

FIG. 7. Dispersion relation of the electron in the ground state (solid line), first excited state (dotted line), and second excited state (dashed line), respectively, as a function of the momentum parallel to the helium surface $K_{y}$, in the presence of an external in-plane magnetic field $B_{\|}=1.5 \mathrm{~T}$ and at the temperature $T=1.3 \mathrm{~K}$, without an external electric field, with $a_{0}$ the Bohr radius.

ous section, which were defined through their response to a parallel magnetic field.

Considering only the ground state, in Fig. 10 we show the behavior of the tunneling probability as a function of time, for different values of the external electric field. As the field intensity increases the tunneling time decreases. The electron-helium surface bound-state lifetime can also be studied by the projection of the wave function at time $t$ on

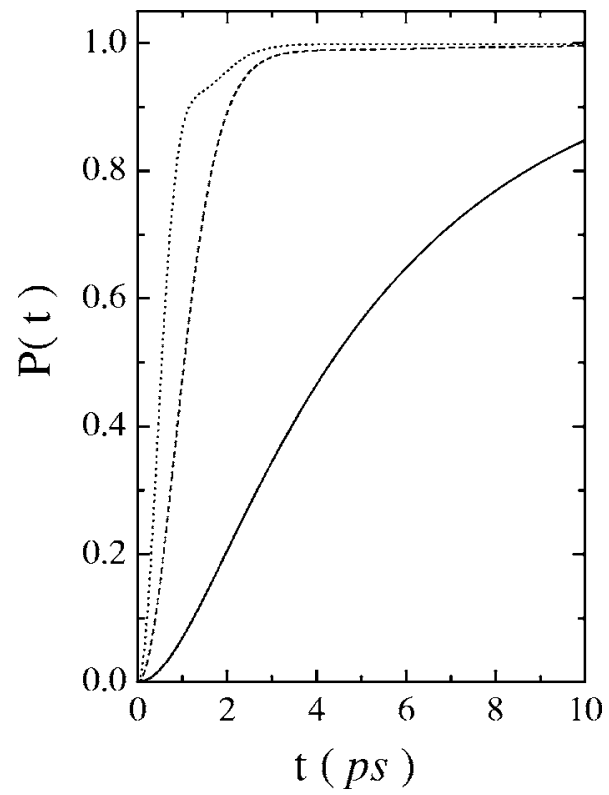

FIG. 8. Tunnel probability $P(t)$ as a function of time for $T$ $=1.0 \mathrm{~K}$ and $F=300 \mathrm{~V} / \mathrm{cm}$. Solid line: ground state; dashed line: first excited state; dotted line: second excited state. 


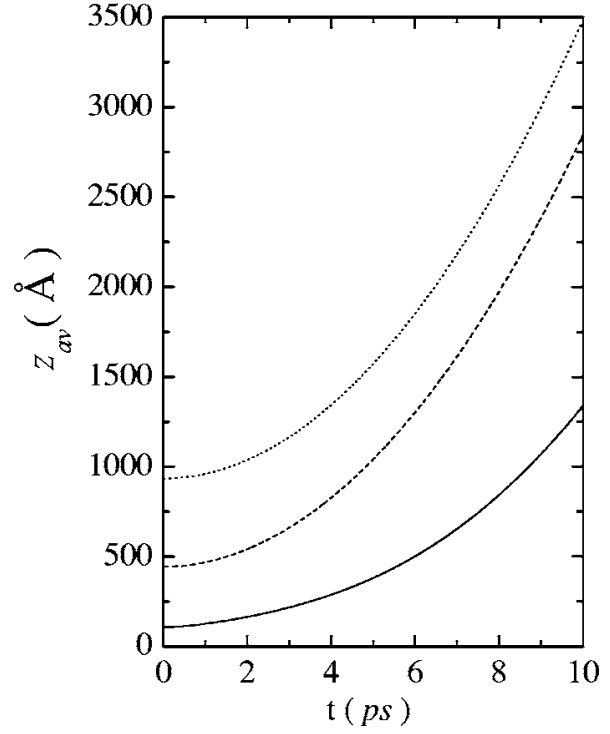

FIG. 9. Average displacement from the interface $z_{a v}$ as a function of time for $T=1.0 \mathrm{~K}$ and $F=300 \mathrm{~V} / \mathrm{cm}$. Solid line: ground state; dashed line: first excited state; dotted line: second excited state.

the initial state $t=0$. We have done this by calculating the electron lifetime for several electric fields and fitting the wave-function projection to an exponential function; that is, $R(t)=\exp (-\Gamma t / \hbar)$. The result for $\Gamma$ is shown in Fig. 11, for the temperature $T=1.0 \mathrm{~K}$. It is clear that $R(t)$ is an exponential function to which $\Gamma(F)$ exhibits a linear dependence with two slopes with crossing point at the electric field $F$ $=700 \mathrm{~V} / \mathrm{cm}$. Below $700 \mathrm{~V} / \mathrm{cm}$ we found $d \Gamma / d F=3.87$ $\times 10^{-4} \mathrm{meV} /(\mathrm{V} / \mathrm{cm})$, and above this electric field we found

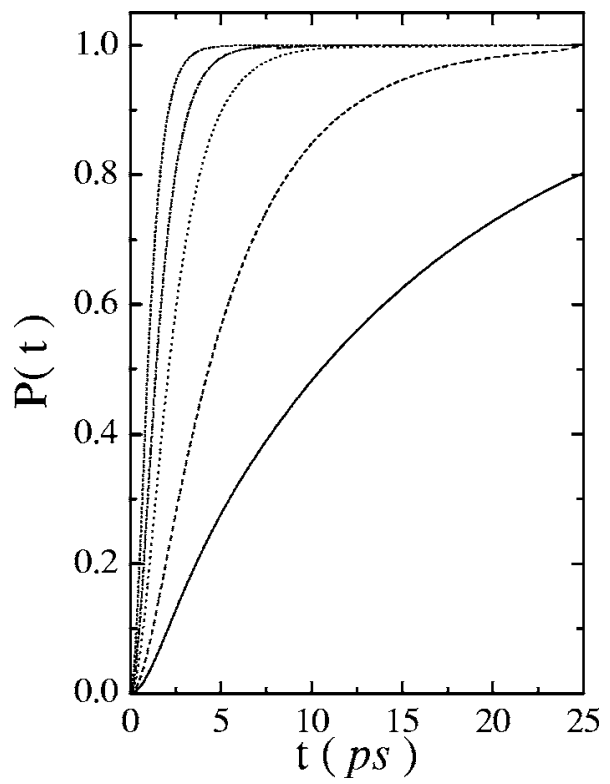

FIG. 10. Tunnel probability $P(t)$ of the ground state as a function of time for $T=1.0 \mathrm{~K}$, for the external electric field: $F$ $=200 \mathrm{~V} / \mathrm{cm}$ (solid line), $F=300 \mathrm{~V} / \mathrm{cm}$ (dashed line), $F$ $=500 \mathrm{~V} / \mathrm{cm}$ (dotted line), $F=700 \mathrm{~V} / \mathrm{cm}$ (dashed-dotted line), and $F=1000 \mathrm{~V} / \mathrm{cm}$ (short-dashed line).

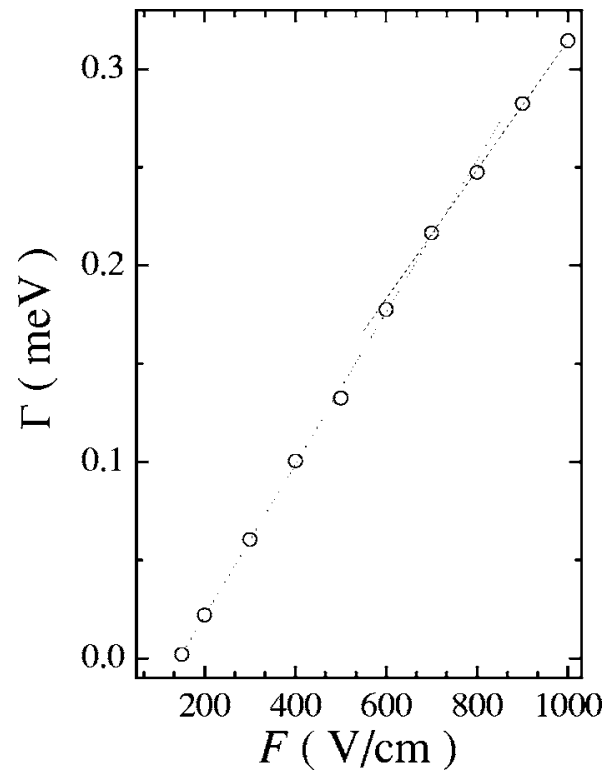

FIG. 11. Electron-helium surface bound-state lifetime of the ground state as a function of the external electric field for $T$ $=1.0 \mathrm{~K}$. The curves are linear fits.

$d \Gamma / d F=3.29 \times 10^{-4} \mathrm{meV} /(\mathrm{V} / \mathrm{cm})$. Finally, in Fig. 12 we show a typical time evolution of the electron ionization under the action of the electric field, the electron probability density is plotted as a function of the $z$-coordinate for several times. In this case, for an external electric $F=500 \mathrm{~V} / \mathrm{cm}$ and temperature $T=1.0 \mathrm{~K}$, the electron gradually leaves the helium interface and tunnels through the potential barrier to infinity. The electron leaves the barrier after 12 ps.

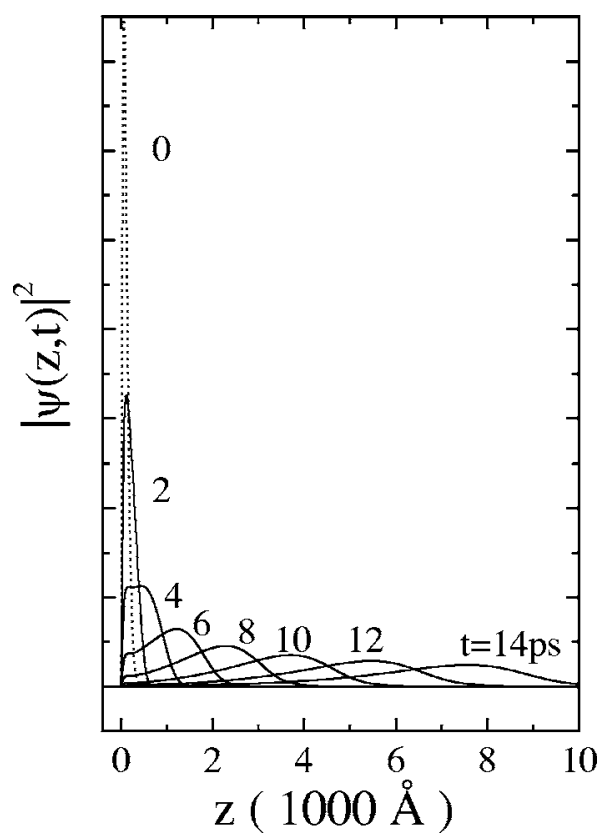

FIG. 12. Time evolution of the ground state wave function when $F=500 \mathrm{~V} / \mathrm{cm}$ and $T=1.0 \mathrm{~K}$. 


\section{CONCLUSION}

In this paper we presented an effective potential model with no fitting parameters that takes into account the diffuseness of the liquid-vapor interface [see Eqs. (3) and (4)], and consequently the helium temperature. The transition energies calculated from this potential between the first (ground state) and the two excited states are in excellent agreement with recent experiments. The numerical results for the energies are obtained from the time evolution method, which is not limited to small perturbations, and the time evolution can be made in the imaginary or the real time domain. With imaginary time propagation, we obtained the eigenstates and the eigenvalues of the first three levels. In the presence of an in-plane magnetic field, we obtained a parallel effective mass that is different from the one perpendicular to the helium surface, obtained by using real time propagation. In addition, the evolution method in real-time can be used to calculate the electron tunneling. The tunneling time was determined as a function of the strength of the perpendicular electric field. A linear dependence on the electric field was found with different slopes for $F<700 \mathrm{~V} / \mathrm{cm}$ and $F>700 \mathrm{~V} / \mathrm{cm}$. The calculation presented here is limited to thick helium films; i.e., bulk liquid helium. In order to obtain the effective potential for thin helium films, we must extend Cole's calculation. ${ }^{2}$ However, notice that for thin helium films, the electron-helium surface states depend considerably on the substrate and temperature. ${ }^{22}$ Such a calculation is beyond the scope of the present paper and is postponed for future work.

\section{ACKNOWLEDGMENTS}

M. H. D. and G. A. F. are supported by the Brazilian National Research Council-CNPq. Part of this work was supported by the Flemish Science Foundation (FWO-Vl), and the EU-RTNetwork on "Surface electrons."
*Electronic address: degani@ fisica.ufc.br

†Electronic address: gil@ fisica.ufc.br

Electronic address: francois.peeters@ua.ac.be

${ }^{1}$ W. T. Sommer, Phys. Rev. Lett. 12, 271 (1964).

${ }^{2}$ M. Cole and M. H. Cohen, Phys. Rev. Lett. 23, 1238 (1969).

${ }^{3}$ V. B. Shikin, Sov. Phys. JETP 31, 936 (1970).

${ }^{4}$ F. M. Peeters, in The Physics of Two-Dimensional Electron Gas, edited by J. T. Devresse and F. M. Peeters (Plenum, New York, 1987).

${ }^{5}$ Two-Dimensional Electron Systems, edited by E. Y. Andrei (Kluwer, Dordrecht, 1997).

${ }^{6}$ Yu. Z. Kovdrya, Low Temp. Phys. 29, 77 (2003), and references therein.

${ }^{7}$ J. A. Northby and C. Kim, Physica B 194-196, 1229 (1994).

${ }^{8}$ L. Menna, S. Yucel, and E. Y. Andrei, Phys. Rev. Lett. 70, 2154 (1993).

${ }^{9}$ M. W. Cole, Phys. Rev. A 1, 1838 (1970).

${ }^{10}$ M. W. Cole, Phys. Rev. B 2, 4239 (1970).

${ }^{11}$ C. C. Grimes, T. R. Brown, M. L. Burns, and C. L. Zipfel, Phys. Rev. B 13, 140 (1976).
${ }^{12}$ O. Hipólito, J. R. D. de Felicio, and G. A. Farias, Solid State Commun. 28, 365 (1978).

${ }^{13}$ F. Stern, Phys. Rev. B 17, 5009 (1978).

${ }^{14}$ M. V. Rama Krishna and K. B. Whaley, Phys. Rev. B 38, 11839 (1988).

${ }^{15}$ E. Cheng, M. W. Cole, and M. H. Cohen, Phys. Rev. B 50, 1136 (1994).

${ }^{16}$ F. Ancilotto and F. Toigo, Phys. Rev. B 50, 12820 (1994).

${ }^{17}$ E. Collin, W. Bailey, P. Fozooni, P. G. Frayne, P. Glasson, K. Harrabi, M. J. Lea, and G. Papageorgiou, Phys. Rev. Lett. 89, 245301 (2002).

${ }^{18}$ M. H. Degani, Appl. Phys. Lett. 59, 57 (1991).

${ }^{19}$ M. H. Degani and J.-P. Leburton, Phys. Rev. B 44, 10901 (1991).

${ }^{20}$ M. H. Degani, Phys. Rev. B 66, 233306 (2002).

${ }^{21}$ W. H. Press, B. P. Flannery, S. A. Teukolsky, and W. T. Vetterling, in Numerical Recipes-The Art of Scientific Computing (Cambridge University Press, Cambridge, 1989), p. 642.

${ }^{22}$ D. Cieslikowskia, A. J. Dahm, and P. Leiderer, Phys. Rev. Lett. 58, 1751 (1987). 